\title{
Experimental Observation of Dynamic-state Switching in VCSELs with Optical Feedback
}

Zhang, Tong; Jia, Zhiwei; Wang, Anbang; Hong, Yanhua; Wang, Longsheng; Guo, Yuanyuan; Wang, Yuncai

\section{IEEE Photonics Technology Letters}

DOI:

10.1109/LPT.2021.3062439

Published: 01/04/2021

Peer reviewed version

Cyswllt i'r cyhoeddiad / Link to publication

Dyfyniad o'r fersiwn a gyhoeddwyd / Citation for published version (APA):

Zhang, T., Jia, Z., Wang, A., Hong, Y., Wang, L., Guo, Y., \& Wang, Y. (2021). Experimental Observation of Dynamic-state Switching in VCSELs with Optical Feedback. IEEE Photonics Technology Letters, 33(7), 335-338. https://doi.org/10.1109/LPT.2021.3062439

\section{Hawliau Cyffredinol / General rights}

Copyright and moral rights for the publications made accessible in the public portal are retained by the authors and/or other copyright owners and it is a condition of accessing publications that users recognise and abide by the legal requirements associated with these rights.

- Users may download and print one copy of any publication from the public portal for the purpose of private study or research.

- You may not further distribute the material or use it for any profit-making activity or commercial gain

- You may freely distribute the URL identifying the publication in the public portal ?

Take down policy

If you believe that this document breaches copyright please contact us providing details, and we will remove access to the work immediately and investigate your claim. 


\title{
Experimental Observation of Dynamic-state Switching in VCSELs with Optical Feedback
}

\author{
Tong Zhang, Zhiwei Jia, Anbang Wang, Member, IEEE, Yanhua Hong, Longsheng Wang, Yuanyuan \\ Guo, and Yuncai Wang
}

\begin{abstract}
Two new dynamic-state switching phenomena are experimentally observed in a vertical-cavity surface-emitting laser with polarization-preserved external-cavity optical feedback. One is switching between a steady state and a quasi-periodic state, and the other is switching between two different steady states. Both switching phenomena occur in the same polarization and the switching period is equal to the round-trip time in the external feedback cavity. The evolution of the two switching phenomena is experimentally investigated in detail. This work not only enriches the understanding of laser nonlinear dynamics but also provides an all-optical alternative for generation of special signals for possible applications.
\end{abstract}

Index Terms - Semiconductor lasers, Vertical-cavity surfaceemitting lasers, Nonlinear dynamics, Dynamic-state switching

\section{INTRODUCTION}

$\mathrm{S}_{\mathrm{fe}}^{\mathrm{E}}$ EMICONDUCTOR lasers with external-cavity optical feedback are ideal objects for studying laser nonlinear dynamics, including chaos, periodic oscillation, and regular pulse package, which can find applications in such diverse fields as secure chaos communication [1]-[3], physical random bit generation [4], [5], chaos range finding [6], and millimeter wave generation [7]. Ridge-waveguide edge-emitting lasers (RWEELs) are the most widely used commercial semiconductor lasers, and RWEELs with external-cavity optical feedback have been well studied [8], [9].

Since its invention, the vertical-cavity surface-emitting laser (VCSEL) has attracted considerable attention due to low threshold, natural single wavelength operation, circular output beam, low cost, etc. [10]. Many studies about dynamics of VCSELs with external-cavity optical feedback have been undertaken. With polarization-preserved or polarizationselective optical feedback, VCSELs can exhibit similar

Manuscript received ; revised; accepted. Date of publication; date of current version. This work was supported in part by the National Natural Science Foundation of China under Grants 61822509, 61731014, 61927811, and 61961136002, in part by the Shanxi Talent Program under Grant 201805D211027, in part by Shanxi "1331 Project" Key Innovative Research Team, in part by the Program for the Top Young and Middle-aged Innovative Talents of Higher Learning Institutions of Shanxi, in part by the Program for Guangdong Introducing Innovative and Entrepreneurial Teams, in part by the International Cooperation of Key R\&D Program of Shanxi Province under Grant 201903D421012. (Corresponding author: Anbang Wang.)

T. Zhang, Z. Jia, A. Wang, L. Wang, and Y. Guo are with the Key Laboratory of Advanced Transducers and Intelligent Control System, Ministry of Education and Shanxi Province, and College of Physics and Optoelectronics, dynamics like RWEELs, such as rapid mode hopping between two adjacent external-cavity modes [11], low frequency fluctuations [12]-[14], regular pulse package [15], and chaos [11], [16]-[17]. Furthermore, due to two linear polarization modes [18], [19], VCSELs with external-cavity optical feedback can exhibit complex dynamics. The existence of two different types of low-frequency fluctuations in VCSELs with external-cavity optical feedback was demonstrated [13]. It was also experimentally found that polarization-preserved feedback can induce random polarization mode hopping [20], [21]. In addition, polarization mode switching was often observed. For example, Li et al. found that $90^{\circ}$ polarization-rotating feedback leads to square-wave polarization switching dynamics for a long external cavity and sinusoidal-wave polarization switching for a short external cavity [22] -[24].

In this letter, two new dynamic-state switching phenomena in a VCSEL with polarization-preserved external-cavity optical feedback have been experimentally observed. One is switching between a steady state and a quasi-periodic state and the other is switching between two different steady states, named S-QP switching and S-S switching, respectively. Both switching phenomena occur in the same polarization with the switching period equal to the round-trip time in the external feedback cavity. We experimentally investigate the evolution of these two new dynamic-state switching phenomena as bias current and feedback strength vary. This work inspires further research on the switching mechanism in semiconductor lasers and enriches the scientific understanding of laser nonlinear dynamics. The two switching phenomena also provide an alloptical alternative for generation of special signals for optical digital signal processing and clock generation, such as dutycycle tunable square-wave modulated photonic microwave signals.

Taiyuan University of Technology, Taiyuan 030024, China (e-mail: zt695241918@live.com; jiazhiwei@tyut.edu.cn; wanganbang@tyut.edu.cn; wanglongsheng@tyut.edu.cn; guoyuanyuan@tyut.edu.cn).

Y. Hong is with the School of Computer Science and Electronic Engineering, Bangor University, Bangor LL57 1UT, U.K. (e-mail: y.hong@bangor.ac.uk).

Y. Wang is with the School of Information Engineering, Guangdong University of Technology, Guangzhou 510006, China (e-mail: wangyc@gdut.edu.cn).

Color versions of one or more of the figures in this letter are available online at.

Digital Object Identifier:.

Copyright (c) 2019 IEEE. Personal use of this material is permitted. 
(a) Experiment setup

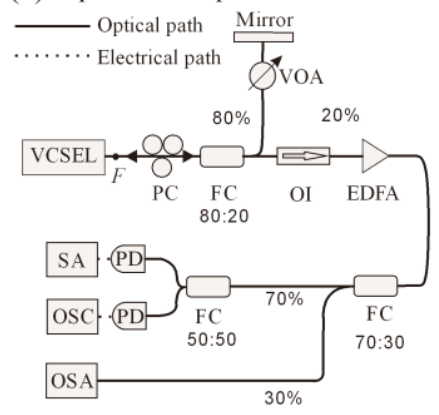

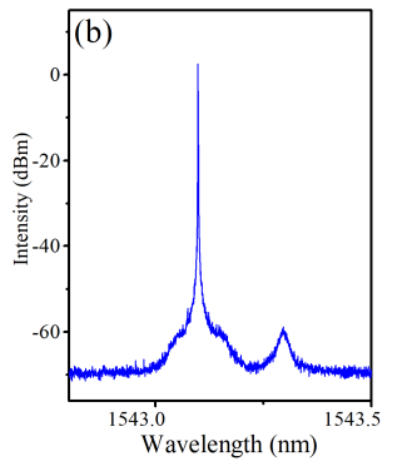

Fig. 1. (a) Experimental setup. PC, polarization controller; FC, fiber coupler; VOA, variable optical attenuator; OI, optical isolator; EDFA, erbium-doped fiber amplifier; PD, photodetector; SA, spectrum analyzer; OSC, oscilloscope; OSA, optical spectrum analyzer. (b) Optical spectrum of the free running VCSEL at bias current $I=4 I_{\text {th }}$.

\section{EXPERIMENTAL SETUP}

The experiment setup of the VCSEL with polarizationpreserved external-cavity optical feedback is shown in Fig. 1(a). The laser (VERTILAS, laser output by fiber coupling, at wavelength of $1550 \mathrm{~nm}$ band) is driven by a low-noise current source (ILX Lightwave LDX 3412), and its working temperature is stabilized at $25.0^{\circ} \mathrm{C}$ by a temperature controller (ILX Lightwave LDT-5416). The output of the laser is divided into two beams by an $80: 20$ fiber coupler (FC). The $80 \%$ beam is directed to a fiber optic mirror and then reflected back into the laser cavity. The laser output facet and the mirror form the external cavity. In this cavity, a polarization controller (PC) is used to match the polarization of the feedback light with the laser, and a variable optical attenuator (VOA) is used to adjust the feedback power. The $20 \%$ beam passes through an optical isolator (OI) and an erbium-doped fiber amplifier (EDFA), and then split into three paths by two couplers for measurement. The VCSEL's optical spectrum is measured by an optical spectrum analyzer (APEX, AP2041B) with a resolution of $0.04 \mathrm{pm}$. The temporal waveform and the power spectrum of the laser intensity are measured by a real-time oscilloscope (LeCroy LABMASTER10ZI, $36 \mathrm{GHz}$ bandwidth) and a spectrum analyzer (Agilent N9020A, $26.5 \mathrm{GHz}$ bandwidth) with $40 \mathrm{GHz}$ photodetectors (Finisar XPDV2120RA-VE-FP).

The free running VCSEL has a threshold current $I_{\text {th }}=1.1 \mathrm{~mA}$ and its optical spectrum at $4 I_{\text {th }}$ is shown in Fig. 1(b). There are two linear polarization (LP) modes, the dominant y-LP mode at $1543.098 \mathrm{~nm}$ and the $\mathrm{x}$-LP mode at $1543.295 \mathrm{~nm}$ with a polarization mode suppression ratio of $61.44 \mathrm{~dB}$ and a wavelength difference of $0.197 \mathrm{~nm}$. The output of the laser stays in y-LP mode as bias current $I$ increases to $9 I_{\text {th }}$. The external-cavity length is about $9.26 \mathrm{~m}$, corresponding to a round-trip time $\tau=92.6 \mathrm{~ns}$ and an external cavity frequency $f_{\mathrm{EC}}$ $=10.8 \mathrm{MHz}$. The feedback strength $\kappa_{\mathrm{f}}$ is defined as the ratio of the feedback power to the laser output power. Due to the unknown coupling loss, the actual feedback strength is smaller than the measured value.

\section{EXPERIMENTAL RESULTS}

\section{A. Switching Between Steady and Quasi-Periodic State (S- QP Switching)}

S-QP switching was observed when the VCSEL was driven by a bias current from $3.7 I_{\text {th }}$ to $6 I_{\text {th }}$. Figure 2 demonstrates a typical S-QP switching obtained at $I=4 I_{\text {th }}$ and $\kappa_{\mathrm{f}}=0.056$. As shown in Fig. 2(a), the laser has a comb-like optical spectrum with a center mode $v_{\mathrm{q}}$ at $1543.107 \mathrm{~nm}$ and side modes with a frequency spacing of $f_{\mathrm{q}}=6.95 \mathrm{GHz}$, which is slightly lower than the relaxation oscillation frequency. Caused by feedback, the optical frequency of the center mode has a slight red shift of $-1.11 \mathrm{GHz}$ relative to the free-running $\mathrm{y}$-LP mode and the $\mathrm{x}-\mathrm{LP}$ also has a slight red shift of $-1.59 \mathrm{GHz}$. Interestingly there is a shorter spectral line, denoted as $v_{\mathrm{s}}$, near the center mode on the long-wavelength side with a frequency difference of -2.72 GHz. From Fig. 2(b), the electrical spectrum has only one highfrequency component at $f_{\mathrm{q}}$ corresponding to the comb spacing, but no oscillation at $2.72 \mathrm{GHz}$ corresponding to the beat frequency between modes $v_{\mathrm{q}}$ and $v_{\mathrm{s}}$. This means that modes $v_{\mathrm{s}}$ and $v_{\mathrm{q}}$ do not exist simultaneously. In addition, as the insert of Fig. 2(b) shows, the spectrum in the low-frequency band has a few spectral lines with an interval equal to $f_{\mathrm{EC}}$. This indicates that the modes $v_{\mathrm{s}}$ and $v_{\mathrm{q}}$ switch with a period equal to the external-cavity round-trip time $\tau$. Figure 2(c) and 2(d) plot the temporal waveform of the laser intensity on different time scales. Clearly, there are two different states switching back and forth with a period of $\tau$. One is the quasi-periodic oscillation with a large amplitude of about $15 \mathrm{mV}$, the other is the steady state with a fixed power. Note that the noise waveform is attributed to the detection noise. Therefore, this switching occurs between a steady state at the optical frequency $v_{\mathrm{s}}$ and a quasi-periodic oscillation at the optical frequency $v_{\mathrm{q}}$.
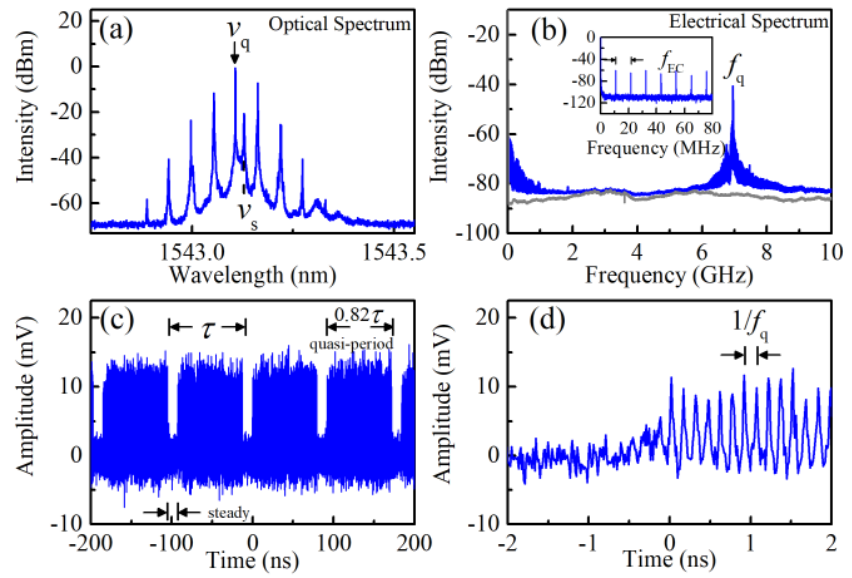

Fig. 2. Switching between steady state and quasi-periodic state at $I=4 I_{\mathrm{th}}, \kappa_{\mathrm{f}}=$ 0.056: (a) optical spectrum, (b) electrical spectrum, (c) and (d) intensity waveforms on different time scales.

Figure 3 shows the evolution of the S-QP switching as feedback strength increases measured at a bias current of $4 I_{\text {th }}$. Under this bias current, the laser changes from a steady state to the S-QP dynamics when the feedback strength exceeds 0.032 . From the first column, one can roughly find that the duty cycle and average amplitude of the quasi-periodic (QP) oscillation increase. Figure 4(a1) plots the duty cycle and average 

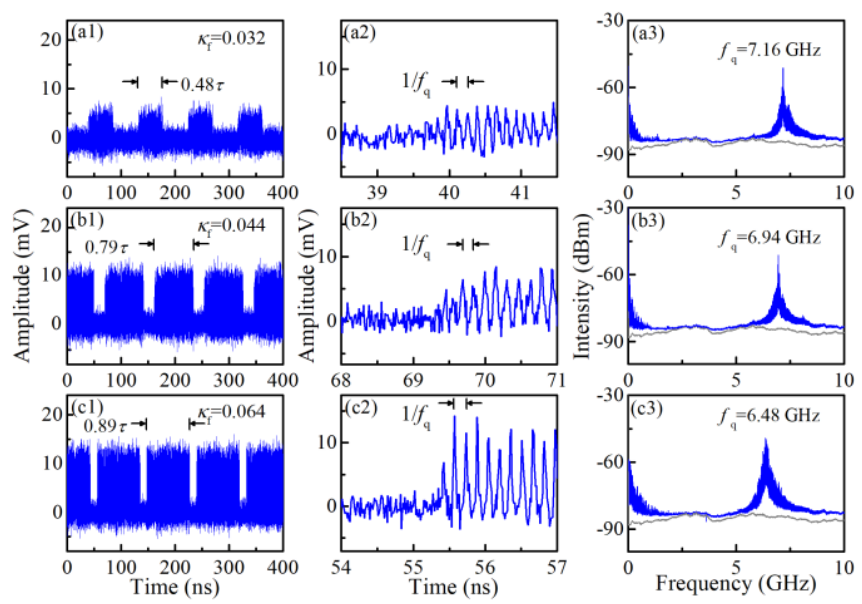

Fig. 3. Switching between steady state and quasi-periodic state at feedback strength of $\kappa_{\mathrm{f}}=0.032,0.044$ and 0.064 , from top to bottom, with the bias current of $I=4 I_{\text {th. }}$. From left to right, columns 1 and 2 are temporal waveforms on different time scale, and column 3 lists electrical spectra.

amplitude of QP oscillation as functions of feedback strength. Shown in Fig. 4(a1), as feedback strength rises to 0.072, the duty cycle increases from 0.48 to 1 , and the average amplitude increases from $6.65 \mathrm{mV}$ to $16.1 \mathrm{mV}$. Further increase of feedback strength leads to a complete QP oscillation, namely the duty cycle is 1 . Obviously, S-QP switching is the transition from steady state to complete QP state. It is worth noting that a similar S-QP switching was recently also found in a distributedfeedback (DFB) semiconductor laser with optical feedback [25], but its evolution is different from that in the VCSEL reported in this work. For the DFB laser, the duty cycle of QP oscillation first increases and then decreases to zero as feedback strength increases, and therefore the S-QP switching will finally evolve into a steady state. By contrast, in the VCSEL, it evolves from the S-QP switching state to a complete QP state. Moreover, as shown in Fig. 4(a2), both the duty cycle and the average amplitude decrease as bias current increases when the feedback strength is fixed. But for the DFB laser [25], the duty cycle increases as bias current increases.

Back to Fig. 3, in the second and third column, we can find that the waveform of the QP oscillation gradually changes from
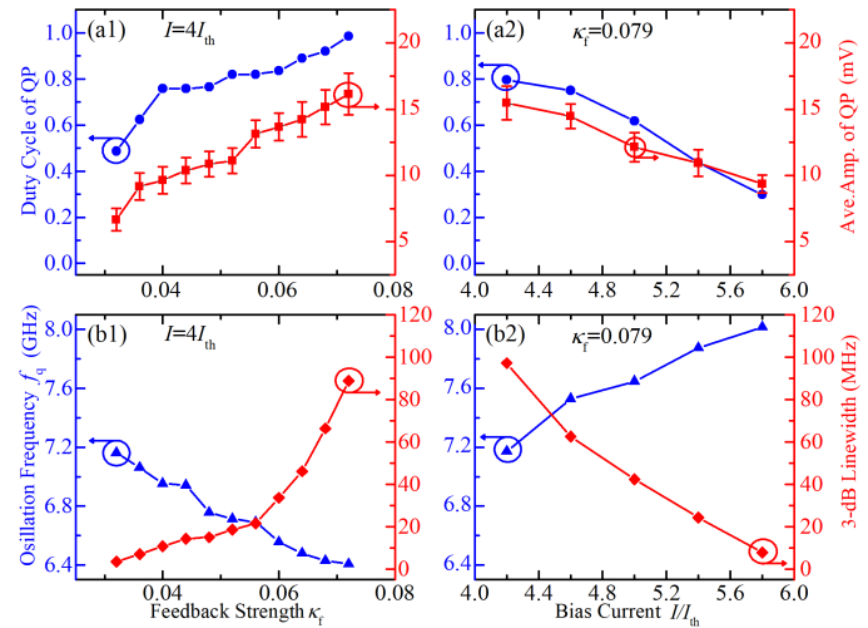

Fig. 4. (a1),(a2) Duty cycle and average amplitude of QP, (b1),(b2) oscillation frequency $f_{\mathrm{q}}$ and 3-dB linewidth of the $f_{\mathrm{q}}$ peak in S-QP switching as function of feedback strength $\kappa_{\mathrm{f}}$ (left column at $I=4 I_{\text {th }}$ ) and bias current (right column at $\left.\kappa_{\mathrm{f}}=0.079\right)$. sinusoidal to pulse-like, and thus the linewidth of the QP spectral line is broadened as feedback strength increases. Figure 4(b1) shows the effects of feedback strength on the QP oscillation frequency and its 3 -dB linewidth at $I=4 I_{\text {th }}$. The oscillation frequency $f_{\mathrm{q}}$ reduces slightly as feedback strength rises. By contrast, the 3-dB linewidth broadens from $3.5 \mathrm{MHz}$ to $88.75 \mathrm{MHz}$. As shown in Fig. 4(b2), the oscillation frequency increases but the $3-\mathrm{dB}$ linewidth decreases with increasing bias current at the fixed feedback strength. The quasi-periodic oscillation originates from the relaxation oscillation. A higher bias current brings a higher relaxation frequency with a larger damping factor, which leads to the results in Fig. 4(b2).

\section{B. Switching Between Two Steady States (S-S Switching)}

As the bias current increases from $6 I_{\text {th }}$ to $9 I_{\text {th }}$, the S-S switching occurs when $\kappa_{\mathrm{f}}$ is less than 0.04. Figure 5 shows a typical S-S switching obtained at $I=7 I_{\text {th }}$ and $\kappa_{\mathrm{f}}=0.0146$. Typically, the optical spectrum as shown in Fig. 5(a) has two spectral lines, $v_{\mathrm{s} 1}$ at $1544.258 \mathrm{~nm}$ and $v_{\mathrm{s} 2}$ at $1544.278 \mathrm{~nm}$ with a frequency difference of $2.58 \mathrm{GHz}$. The power of the shortwavelength mode is slightly higher than the long-wavelength mode. The electrical spectrum of the laser output in Fig. 5(b) does not have a peak at $2.58 \mathrm{GHz}$. This means that the two modes do not exist simultaneously. In addition, as shown in Fig. 5(c), the electrical spectrum in the low-frequency band has a few spectral lines with an interval equal to $f_{\mathrm{EC}}$. This indicates that the two modes are steady states and switch with a period of $\tau$. Figure 5(d) shows the temporal waveform of the S-S switching which is measured by the oscilloscope with DC coupling. The output of the VCSEL switches regularly between two different steady states with a period of $\tau$. The steady state with higher level corresponds to the short-wavelength mode, and the steady state with lower level is the long-wavelength mode.
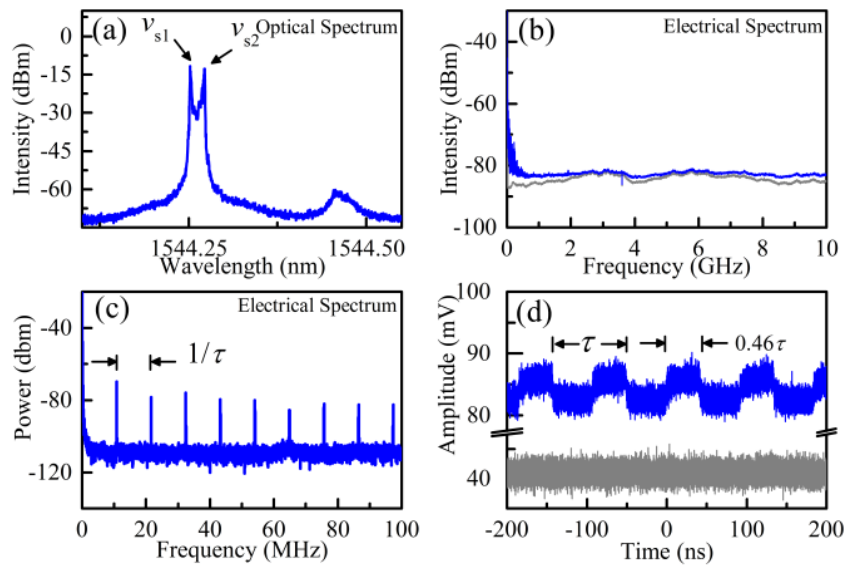

Fig. 5. Switching between two steady states at $I=7 I_{\mathrm{th}}, \kappa_{\mathrm{f}}=0.0146$ : (a) optical spectrum, (b) electrical spectrum, (c) low-frequency-band electrical spectrum, and (d) temporal waveform. The gray lower waveform is background noise of the detector.

Figure 6 shows the duty cycle of the high-level steady state and the wavelengths of the two modes as functions of the feedback strength at $I=7 I_{\text {th }}$. S-S switching, characterized by the duty cycle between 0 and 1 and two-peak optical spectra, appears when $\kappa_{\mathrm{f}}$ is in the range of $0.0017 \sim 0.037$. As shown in Fig.6, as feedback strength increases in this range, the duty 


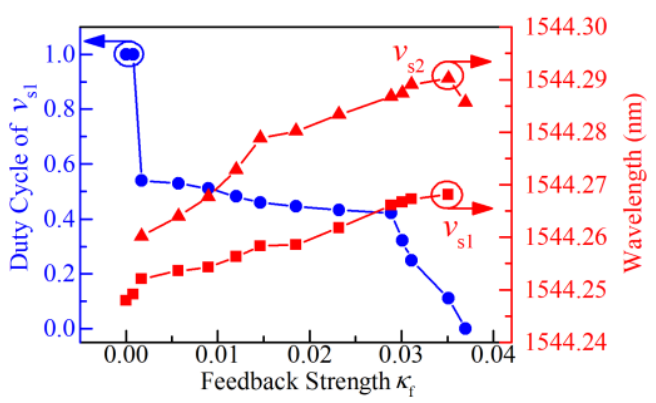

Fig. 6. Duty cycle of $v_{\mathrm{s} 1}$ and wavelength of $v_{\mathrm{s} 1}$ and $v_{\mathrm{s} 2}$ versus the feedback strength $\kappa_{\mathrm{f}}$ when the laser switches between two steady states at a bias current of $I=7 I_{\mathrm{th}}$.

cycle of $v_{\mathrm{s} 1}$ decreases from 1 to 0 and the laser output changes into the steady state at the long wavelength. In addition, as $\kappa_{\mathrm{f}}$ increases, both $v_{\mathrm{s} 1}$ and $v_{\mathrm{s} 2}$ red shift. The fluctuation of the refractive index, which results from the carrier dynamics, is more intense in S-S switching than that in single steady state, leading to slightly higher index. As the result, $v_{\mathrm{s} 1}$ rapidly red shifts when laser output changes into S-S switching, and $v_{\mathrm{s} 2}$ slightly blue shifts when laser output changes out from S-S switching.

Compared with the S-QP switching, the S-S switching appears at higher bias current and a lower feedback strength. In this condition, the laser has a larger damping factor and its relaxation oscillation cannot be forced into undamping to generate periodic or QP oscillation by the weak optical feedback.

\section{CONCLUSION}

In conclusion, two new dynamic-state switching phenomena are experimentally found in the VCSEL with optical feedback. One is the switching between a steady state and a quasi-periodic state, which is the transition from a steady to a complete quasiperiodic state. As bias current increases and feedback strength decreases, the other one occurs, i.e., switching between two steady states with an optical frequency difference of a few $\mathrm{GHz}$. After the S-S switching, the laser will turn back to a steady state but with different optical frequency. The switching period is equal to the round-trip time in the external cavity. These dynamic-state switching phenomena can enrich the understanding of the dynamics of semiconductor lasers with optical feedback. Furthermore, they provide an alternative for generation of square-wave photonic microwave signals which is useful in signal processing and communication systems.

\section{REFERENCES}

[1] L. M. Pecora and T. L. Carroll, "Synchronization in chaotic systems," Phys. Rev. Lett., vol. 64, no. 8, pp. 821-824, Feb. 1990.

[2] J. Ohtsubo, "Chaos synchronization and chaotic signal masking in semiconductor lasers with optical feedback," IEEE J. Quantum Electron., vol. 38, no. 9, pp. 1141-1154, Sep. 2002

[3] M. Sciamanna and K. A. Shore, "Physics and applications of laser diode chaos," Nat. Photonics, vol. 9, no. 3, pp. 151-162, Feb. 2015.

[4] A. Uchida et al., "Fast physical random bit generation with chaotic semiconductor lasers," Nat. Photonics, vol. 2, no. 12, pp. 728-732, Nov. 2008.

[5] I. Kanter, Y. Aviad, I. Reidler, E. Cohen, and M. Rosenbluh, "An optical ultrafast random bit generator," Nat. Photonics, vol. 4, no. 1, pp. 58-61, Dec. 2009

[6] C. Y. Chen, C. H. Cheng, D. K. Pan, and F. Y. Lin, "Experimental generation and analysis of chaos-modulated pulses for pulsed chaos lidar applications based on gain-switched semiconductor lasers subject to optical feedback," Opt. Express, vol. 26, no. 16, pp. 20851-20860, Aug. 2018.

[7] J. W. Wu, B. Nakarmi, and Y. H. Won, "Optically tunable microwave, millimeter-wave and submillimeter-wave utilizing single-mode FabryPerot laser diode subject to optical feedback," Opt. Express, vol. 24, no. 3, pp. 2655-2663, Feb. 2016.

[8] A. Wang, Y. Wang, and H. He, "Enhancing the Bandwidth of the Optical Chaotic Signal Generated by a Semiconductor Laser with Optical Feedback," IEEE Photonics Technol. Lett., vol. 20, no. 19, pp. 1633-1635, Oct. 2008.

[9] C. Masoller, "Anticipation in the Synchronization of Chaotic Semiconductor Lasers with Optical Feedback," Phys. Rev. Lett., vol. 86, no. 13, pp. 2782-2785, Mar. 2001.

[10] A. Liu, P. Wolf, J. A. Lott, and D. Bimberg, "Vertical-cavity surfaceemitting lasers for data communication and sensing," Photonics Res., vol. 7, no. 2, pp. 121-136, Feb. 2019.

[11] Y. C. Chung and Y. H. Lee, "Spectral characteristics of vertical-cavity surface-emitting lasers with external optical feedback," IEEE Photo. Technol. Lett., vol. 3, no. 7, pp. 597-599, Jul. 1991.

[12] C. Masoller and N. B. Abraham, "Low-frequency fluctuations in verticalcavity surface-emitting semiconductor lasers with optical feedback," Phys. Rev. A, vol. 59, no. 4, pp. 3021-3031, Apr. 1999.

[13] M. Sciamanna, C. Masoller, N. B. Abraham, F. Rogister, P. Mégret, and M. Blondel, "Different regimes of low-frequency fluctuations in verticalcavity surface-emitting lasers," J. Opt. Soc. Am. B, vol. 20, no. 1, pp. 3744, Jan. 2003.

[14] M. C. Soriano et al., "Low-Frequency Fluctuations in Vertical-Cavity Surface-Emitting Lasers with Polarization Selective Feedback: Experiment and Theory," IEEE J. Sel. Top. Quantum Electron., vol. 10, no. 5, pp. 998-1005, Sep. 2004.

[15] A. Tabaka et al., "Dynamics of vertical-cavity surface-emitting lasers in the short external cavity regime: Pulse packages and polarization mode competition," Phys. Rev. A, vol. 73, no. 1, pp. 013810, Jan. 2006.

[16] J. Y. Law and G. P. Agrawal, "Effects of optical feedback on static and dynamic characteristics of vertical-cavity surface-emitting lasers," IEEE J. Sel. Top. Quantum Electron., vol. 3, no. 2, pp. 353-358, Apr. 1997.

[17] H. Aoyama, S. Tomida, R. Shogenji, and J. Ohtsubo, "Chaos dynamics in vertical-cavity surface-emitting semiconductor lasers with polarizationselected optical feedback," Opt. Commun., vol. 284, no. 5, pp. 1405-1411, Oct. 2011.

[18] C. Masoller and M. S. Torre, "Influence of optical feedback on the polarization switching of vertical-cavity surface-emitting lasers," IEEE J. Quantum Electron., vol. 41, no. 4, pp. 483-489, Apr. 2005.

[19] Y. Hong, P. S. Spencer, and K. A. Shore, "Suppression of polarization switching in vertical-cavity surface-emitting lasers by use of optical feedback," Opt. Lett., vol. 29, no. 18, pp. 2151-2153, Sep. 2004.

[20] M. Sciamanna, K. Panajotov, H. Thienpont, I. Veretennicoff, P. Megret, and M. Blondel, "Optical feedback induces polarization mode hopping in vertical-cavity surface-emitting lasers," Opt. Lett., vol. 28, no. 17, pp. 1543-1545, Sep. 2003.

[21] K. Panajotov et al., "Residence time distribution and coherence resonance of optical-feedback-induced polarization mode hopping in vertical-cavity surface-emitting lasers," Phys. Rev. A, vol. 69, no. 1, pp. 011801, Jan. 2004.

[22] H. Li, A. Hohl, A. Gavrielides, H. Hou, and K. D. Choquette, "Stable polarization self-modulation in vertical-cavity surface-emitting lasers," Appl. Phys. Lett., vol. 72, no. 19, pp. 2355-2357, May 1998.

[23] D. W. Sukow, T. Gilfillan, B. Pope, M. S. Torre, A. Gavrielides, and C. Masoller, "Square-wave switching in vertical-cavity surface-emitting lasers with polarization-rotated optical feedback: Experiments and simulations," Phys. Rev. A, vol. 86, no. pp. 033818, Sep. 2012.

[24] J. Mulet, M. Giudici, J. Javaloyes, and S. Balle, "Square-wave switching by crossed-polarization gain modulation in vertical-cavity semiconductor lasers," Phys. Rev. A, vol. 76, no. 4, pp. 043801, Oct. 2007

[25] J. X. Dong, J. P. Zhuang, and S. C. Chan, "Tunable switching between stable and periodic states in a semiconductor laser with feedback," Opt. Lett., vol. 42, no. 21, pp. 4291-4294, Nov. 2017. 